\title{
OPERATORS ON TWO BANACH SPACES OF CONTINUOUS FUNCTIONS ON LOCALLY COMPACT SPACES OF ORDINALS
}

\author{
TOMASZ KANIA AND NIELS JAKOB LAUSTSEN
}

(Communicated by Thomas Schlumprecht)

\begin{abstract}
Denote by $\left[0, \omega_{1}\right)$ the set of countable ordinals, equipped with the order topology, let $L_{0}$ be the disjoint union of the compact ordinal intervals $[0, \alpha]$ for $\alpha$ countable, and consider the Banach spaces $C_{0}\left[0, \omega_{1}\right)$ and $C_{0}\left(L_{0}\right)$ consisting of all scalar-valued, continuous functions which are defined on the locally compact Hausdorff spaces $\left[0, \omega_{1}\right)$ and $L_{0}$, respectively, and which vanish eventually. Our main result states that a bounded, linear operator $T$ between any pair of these two Banach spaces fixes an isomorphic copy of $C_{0}\left(L_{0}\right)$ if and only if the identity operator on $C_{0}\left(L_{0}\right)$ factors through $T$, if and only if the Szlenk index of $T$ is uncountable. This implies that the set $\mathscr{S}_{C_{0}\left(L_{0}\right)}\left(C_{0}\left(L_{0}\right)\right)$ of $C_{0}\left(L_{0}\right)$-strictly singular operators on $C_{0}\left(L_{0}\right)$ is the unique maximal ideal of the Banach algebra $\mathscr{B}\left(C_{0}\left(L_{0}\right)\right)$ of all bounded, linear operators on $C_{0}\left(L_{0}\right)$, and that $\mathscr{S}_{C_{0}\left(L_{0}\right)}\left(C_{0}\left[0, \omega_{1}\right)\right)$ is the second-largest proper ideal of $\mathscr{B}\left(C_{0}\left[0, \omega_{1}\right)\right)$. Moreover, it follows that the Banach space $C_{0}\left(L_{0}\right)$ is primary and complementably homogeneous.
\end{abstract}

\section{Introduction AND STATEMENT OF MAIN RESUltS}

The purpose of this paper is to advance our understanding of the (bounded, linear) operators acting on the Banach space $C_{0}\left[0, \omega_{1}\right)$ of scalar-valued, continuous functions which vanish eventually and which are defined on the locally compact Hausdorff space $\left[0, \omega_{1}\right)$ of all countable ordinals, equipped with the order topology. Another Banach space of a similar kind, $C_{0}\left(L_{0}\right)$, will play a key role; here $L_{0}$ denotes the locally compact Hausdorff space given by the disjoint union of the compact ordinal intervals $[0, \alpha]$ for $\alpha$ countable or, equivalently,

$$
L_{0}=\bigcup_{\alpha<\omega_{1}}[0, \alpha] \times\{\alpha+1\},
$$

endowed with the topology inherited from the product topology on $\left[0, \omega_{1}\right)^{2}$. As a spin-off of our main line of inquiry, we obtain some conclusions of possible independent interest concerning the Banach space $C_{0}\left(L_{0}\right)$ and the operators acting on it.

The motivation behind this work comes primarily from our previous studies 13 , 14. (the latter written jointly with P. Koszmider) of the Banach algebra $\mathscr{B}\left(C_{0}\left[0, \omega_{1}\right)\right)$ of all operators on $C_{0}\left[0, \omega_{1}\right)$. Indeed, the main theorem of 14 implies that

Received by the editors April 17, 2013 and, in revised form, February 4, 2014.

2010 Mathematics Subject Classification. Primary 46H10, 47B38, 47L10; Secondary 06F30, 46B26, 47L20.

Key words and phrases. Banach algebra, maximal ideal, bounded, linear operator, Szlenk index, continuous function, ordinal interval, order topology, Banach space, primary, complementably homogeneous. 
$\mathscr{B}\left(C_{0}\left[0, \omega_{1}\right)\right)$ has a unique maximal ideal $\mathscr{M}$ (the Loy-Willis ideal), while [13, Theorem 1.6] characterizes this ideal as the set of operators which factor through the Banach space $C_{0}\left(L_{0}\right)$ :

$$
\mathscr{M}=\left\{T S: S \in \mathscr{B}\left(C_{0}\left[0, \omega_{1}\right), C_{0}\left(L_{0}\right)\right), T \in \mathscr{B}\left(C_{0}\left(L_{0}\right), C_{0}\left[0, \omega_{1}\right)\right)\right\},
$$

using the alternative description of $C_{0}\left(L_{0}\right)$ given in Lemma 2.6(i), below.

To state the main result of the present paper precisely, we require the following three pieces of terminology concerning an operator $T$ between the Banach spaces $X$ and $Y$ :

- Let $W$ be a Banach space. Then $T$ fixes an isomorphic copy of $W$ if there exists an operator $U: W \rightarrow X$ such that the composite operator $T U$ is an isomorphism onto its range.

- An operator $V$ between the Banach spaces $W$ and $Z$ factors through $T$ if there exist operators $R: W \rightarrow X$ and $S: Y \rightarrow Z$ such that $V=S T R$.

- Suppose that $X$ is an Asplund space. Then each weakly* compact subset $K$ of the dual space $X^{*}$ of $X$ has associated with it a certain ordinal, which is called the Szlenk index of $K$; we refer to [17] or [12, Section 2.4] for the precise definition of this notion. The Szlenk index of the operator $T$ is then defined as the Szlenk index of the image under the adjoint operator of $T$ of the closed unit ball of $Y^{*}$.

Theorem 1.1. Let $X$ and $Y$ each denote either of the Banach spaces $C_{0}\left[0, \omega_{1}\right)$ and $C_{0}\left(L_{0}\right)$, and let $T$ be an operator from $X$ to $Y$. Then the following three conditions are equivalent:

(a) $T$ fixes an isomorphic copy of $C_{0}\left(L_{0}\right)$;

(b) the identity operator on $C_{0}\left(L_{0}\right)$ factors through $T$;

(c) the Szlenk index of $T$ is uncountable.

In the case where $X=Y \in\left\{C_{0}\left[0, \omega_{1}\right), C_{0}\left(L_{0}\right)\right\}$, Theorem 1.1 leads to significant new information about the lattice of closed ideals of the Banach algebra $\mathscr{B}(X)$ of all operators on $X$. (Throughout this paper, the term ideal means a two-sided, algebraic ideal, while a maximal ideal is a proper ideal which is maximal with respect to inclusion among all proper ideals.) To facilitate the statement of these conclusions, let us introduce the notation $\mathscr{S}_{W}(X)$ for the set of those operators on $X$ that do not fix an isomorphic copy of the Banach space $W$; such operators are called $W$-strictly singular. The set $\mathscr{S}_{W}(X)$ is closed in the norm topology, and it is closed under composition by arbitrary operators, so that $\mathscr{S}_{W}(X)$ is a closed ideal of $\mathscr{B}(X)$ if and only if $\mathscr{S}_{W}(X)$ is closed under addition.

Corollary 1.2. Let $X=C_{0}\left[0, \omega_{1}\right)$ or $X=C_{0}\left(L_{0}\right)$. Then

$$
\begin{aligned}
\mathscr{S}_{C_{0}\left(L_{0}\right)}(X)=\{T \in \mathscr{B}(X): & \\
& \text { the identity operator on } \left.C_{0}\left(L_{0}\right) \text { does not factor through } T\right\} \\
= & \{T \in \mathscr{B}(X): \text { the Szlenk index of } T \text { is countable }\},
\end{aligned}
$$

and this set is a proper closed ideal of $\mathscr{B}(X)$.

In the case where $X=C_{0}\left(L_{0}\right)$, this ideal is the unique maximal ideal of $\mathscr{B}\left(C_{0}\left(L_{0}\right)\right)$, whereas for $X=C_{0}\left[0, \omega_{1}\right)$, this ideal is the second-largest proper ideal 
of $\mathscr{B}\left(C_{0}\left[0, \omega_{1}\right)\right)$, in the following precise sense:

- $\mathscr{S}_{C_{0}\left(L_{0}\right)}\left(C_{0}\left[0, \omega_{1}\right)\right)$ is properly contained in the Loy-Willis ideal $\mathscr{M}$; and

- for each proper ideal $\mathscr{I}$ of $\mathscr{B}\left(C_{0}\left[0, \omega_{1}\right)\right)$, either $\mathscr{I}=\mathscr{M}$ or $\mathscr{I} \subseteq$ $\mathscr{S}_{C_{0}\left(L_{0}\right)}\left(C_{0}\left[0, \omega_{1}\right)\right)$.

Finally, as an easy Banach-space theoretic consequence of these results, we shall show that $C_{0}\left(L_{0}\right)$ has the following two properties, defined for any Banach space $X$ :

- $X$ is primary if, for each projection $P \in \mathscr{B}(X)$, either the kernel of $P$ or the range of $P$ (or both) is isomorphic to $X$;

- $X$ is complementably homogeneous if, for each closed subspace $W$ of $X$ such that $W$ is isomorphic to $X$, there exists a closed, complemented subspace $Y$ of $X$ such that $Y$ is isomorphic to $X$ and $Y$ is contained in $W$.

Corollary 1.3. The Banach space $C_{0}\left(L_{0}\right)$ is primary and complementably homogeneous.

The counterpart of this corollary is true for $C_{0}\left[0, \omega_{1}\right)$; this is due to Alspach and Benyamini [3, Theorem 1] and the present authors and Koszmider [13, Corollary 1.12], respectively.

\section{Preliminaries}

In this section we shall explain our conventions and terminology in further detail, state some important theorems that will be required in the proof of Theorem [1.1. and establish some auxiliary results.

All Banach spaces are supposed to be over the same scalar field, which is either the real field $\mathbb{R}$ or the complex field $\mathbb{C}$. By an operator, we understand a bounded and linear mapping between Banach spaces. We write $I_{X}$ for the identity operator on the Banach space $X$.

The following elementary characterization of the operators that the identity operator factors through is well known.

Lemma 2.1. Let $X, Y$, and $Z$ be Banach spaces. Then the identity operator on $Z$ factors through an operator $T: X \rightarrow Y$ if and only if $X$ contains a closed subspace $W$ such that:

- $W$ is isomorphic to $Z$;

- the restriction of $T$ to $W$ is bounded below, in the sense that there exists a constant $\varepsilon>0$ such that $\|T w\| \geqslant \varepsilon\|w\|$ for each $w \in W$

- the image of $W$ under $T$ is complemented in $Y$.

For an ordinal $\alpha$ and a pair $(X, Y)$ of Banach spaces, let $\mathscr{S} \mathscr{Z}_{\alpha}(X, Y)$ denote the set of operators $T: X \rightarrow Y$ such that the Szlenk index of $T$ is defined and does not exceed $\omega^{\alpha}$. Brooker [8, Theorem 2.2] has proved the following result.

Theorem 2.2 (Brooker). The class $\mathscr{S}_{\mathscr{Z}}{ }_{\alpha}$ is a closed, injective, and surjective operator ideal in the sense of Pietsch for each ordinal $\alpha$.

For a Hausdorff space $K$, we denote by $C(K)$ the vector space of all scalarvalued, continuous functions defined on $K$. In the case where $K$ is locally compact, the subspace $C_{0}(K)$ consisting of those functions $f \in C(K)$ for which the set $\{k \in K:|f(k)| \geqslant \varepsilon\}$ is compact for each $\varepsilon>0$ is a Banach space with respect to the supremum norm, and we have $C(K)=C_{0}(K)$ if and only if $K$ is compact. 
The case where $K$ is an ordinal interval (always equipped with the order topology) will be particularly important for us. Bessaga and Pełczyński 4 have shown that the Banach spaces $C\left[0, \omega^{\omega^{\alpha}}\right]$, where $\alpha$ is a countable ordinal, exhaust all possible isomorphism classes of Banach spaces of the form $C(K)$ for a countably infinite, compact metric space $K$. The Banach spaces $C\left[0, \omega^{\omega^{\alpha}}\right]$ may be viewed as higherordinal analogues of the Banach space $c_{0}$, which corresponds to the case where $\alpha=0$.

The Szlenk index can be used to distinguish these Banach spaces because Samuel [16] has shown that $C\left[0, \omega^{\omega^{\alpha}}\right]$ has Szlenk index $\omega^{\alpha+1}$ for each countable ordinal $\alpha$; a simplified proof of this result, due to Hájek and Lancien [11, is given in [12, Theorem 2.59].

More importantly for our purposes, Bourgain [6] has proved that each operator $T$, defined on a $C(K)$-space and of sufficiently large Szlenk index, fixes an isomorphic copy of $C[0, \alpha]$ for some countable ordinal $\alpha$, which increases with the Szlenk index of $T$. The precise statement of this result is as follows.

Theorem 2.3 (Bourgain). Let $X$ be a Banach space, let $K$ be a compact Hausdorff space, and let $\alpha$ be a countable ordinal. Then each operator $T: C(K) \rightarrow X$ whose Szlenk index exceeds $\omega^{\alpha}$ fixes an isomorphic copy of $C\left[0, \omega^{\omega \cdot \alpha}\right]$.

Remark 2.4. (i) Bourgain first proves Theorem 2.3 in the case where $K$ is a compact metric space [6, Proposition 3], and he then explains how to deduce the result for general $K$ [6, p. 107].

(ii) Alspach [2] has shown that the most obvious strengthening of Bourgain's theorem is false by constructing a surjective operator $T$ on $C\left[0, \omega^{\omega^{2}}\right]$ such that $T$ does not fix an isomorphic copy of $C\left[0, \omega^{\omega^{2}}\right]$. (The surjectivity of $T$ implies that $T$ has the same Szlenk index as its codomain, that is, $\omega^{3}$.)

We shall use Bourgain's theorem in tandem with the following theorem of Pełczyński [15, Theorem 1], which will enable us to infer that the identity operator on $C(K)$, where $K$ is a compact metric space, factors through each operator which fixes an isomorphic copy of $C(K)$ and which has separable codomain.

Theorem 2.5 (Pełczyński). Let $W$ be a closed subspace of a separable Banach space $X$, and suppose that $W$ is isomorphic to $C(K)$ for some compact metric space $K$. Then $W$ contains a closed subspace which is isomorphic to $C(K)$ and which is complemented in $X$.

To conclude this section, we shall state some results about the Banach spaces $C_{0}\left[0, \omega_{1}\right)$ and $C_{0}\left(L_{0}\right)$ that will be required in the proof of Theorem 1.1. As in [13, it turns out to be convenient to work with an alternative representation of the Banach space $C_{0}\left(L_{0}\right)$, stated in Lemma 2.6(i), below. This relies on the following piece of notation. Denote by

$$
\left(\bigoplus_{j \in J} X_{j}\right)_{c_{0}}=\left\{\left(x_{j}\right)_{j \in J}: x_{j} \in X_{j}(j \in J) \text { and }\left\{j \in J:\left\|x_{j}\right\| \geqslant \varepsilon\right\} \text { is finite }(\varepsilon>0)\right\}
$$

the $c_{0}$-direct sum of a family $\left(X_{j}\right)_{j \in J}$ of Banach spaces, set $E_{\omega_{1}}=\left(\bigoplus_{\alpha<\omega_{1}} C[0, \alpha]\right)_{c_{0}}$ and, more generally, $E_{A}=\left(\bigoplus_{\alpha \in A} C[0, \alpha]\right)_{c_{0}}$ for each non-empty subset $A$ of $\left[0, \omega_{1}\right)$. 
Lemma 2.6. (i) The Banach spaces $C_{0}\left(L_{0}\right)$ and $E_{\omega_{1}}$ are isometrically isomorphic.

(ii) The Banach space $E_{\omega_{1}}$ is isomorphic to the $c_{0}$-direct sum of countably many copies of itself: $E_{\omega_{1}} \cong c_{0}\left(\mathbb{N}, E_{\omega_{1}}\right)$.

(iii) Let $A$ be an uncountable subset of $\left[0, \omega_{1}\right)$. Then $E_{A}$ is isomorphic to $E_{\omega_{1}}$.

(iv) The Banach space $C_{0}\left[0, \omega_{1}\right)$ contains a closed, complemented subspace which is isomorphic to $E_{\omega_{1}}$.

(v) The following three conditions are equivalent for each operator $T$ on $C_{0}\left[0, \omega_{1}\right)$ :

(a) $T$ belongs to the Loy-Willis ideal $\mathscr{M}$;

(b) the range of $T$ is contained in a closed, complemented subspace which is isomorphic to $E_{\omega_{1}}$;

(c) the identity operator on $C_{0}\left[0, \omega_{1}\right)$ does not factor through $T$.

Proof. Clause (ii) is a special instance of a well-known elementary fact (see, e.g., [9, p. 191, Exercise 9]), while clauses (iii) and (iv) are [13, Lemma 2.12 and Corollary 2.16], respectively.

To prove clause (iii), we observe that the Banach spaces $E_{\omega_{1}}$ and $E_{A}$ contain complemented copies of each other. By (iii), the Pełczyński decomposition method (as stated in [1, Theorem 2.23(b)], for instance) applies, and hence the conclusion follows.

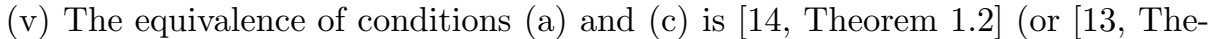
orem 1.6, (i) $\Leftrightarrow($ viii)]), while the equivalence of conditions (ㅁa) and (b-b) follows from [13, Theorem 1.6]; more precisely, (b) implies (可) by [13, Theorem 1.6(iv) $\Rightarrow(\mathrm{i})]$, and the converse can be shown as in the proof of [13, Theorem 1.6 (iii) $\Rightarrow($ iv)].

For each countable ordinal $\alpha$, let $P_{\alpha}$ be the norm-one projection on $C_{0}\left[0, \omega_{1}\right)$ given by $P_{\alpha} f=f \cdot \mathbf{1}_{[0, \alpha]}$ for each $f \in C_{0}\left[0, \omega_{1}\right)$, where $\mathbf{1}_{[0, \alpha]}$ denotes the characteristic function of the ordinal interval $[0, \alpha]$.

Lemma 2.7. (i) A subspace of $C_{0}\left[0, \omega_{1}\right)$ is separable if and only if it is contained in the range of the projection $P_{\alpha}$ for some countable ordinal $\alpha$.

(ii) Let $T$ be an operator from $C_{0}\left[0, \omega_{1}\right)$ into a Banach space $X$. Then $T$ has separable range if and only if $T=T P_{\alpha}$ for some countable ordinal $\alpha$.

Proof. Clause (ii) is a special case of [14, Lemma 4.2].

(iii) The implication $\Leftarrow$ is clear because $P_{\alpha}$ has separable range.

We shall prove the converse by contradiction. Assume that $T$ has separable range and that $T \neq T P_{\alpha}$ for each $\alpha<\omega_{1}$. Since each element of $C_{0}\left[0, \omega_{1}\right)$ has countable support, we may inductively construct a transfinite sequence of disjointly supported functions $\left(f_{\alpha}\right)_{\alpha<\omega_{1}}$ in $C_{0}\left[0, \omega_{1}\right)$ such that $T f_{\alpha} \neq 0$ and $\left\|f_{\alpha}\right\|=1$ for each $\alpha<\omega_{1}$. Set

$$
A(n)=\left\{\alpha \in\left[0, \omega_{1}\right):\left\|T f_{\alpha}\right\| \geqslant \frac{1}{n}\right\} \quad(n \in \mathbb{N}) .
$$

Since $\left[0, \omega_{1}\right)=\bigcup_{n \in \mathbb{N}} A(n)$, we can find $n_{0} \in \mathbb{N}$ such that $A\left(n_{0}\right)$ is uncountable.

Take a sequence $\left(x_{m}\right)_{m \in \mathbb{N}}$ which is dense in the range of $T$, and set

$$
B(m)=\left\{\alpha \in A\left(n_{0}\right):\left\|x_{m}-T f_{\alpha}\right\| \leqslant \frac{1}{3 n_{0}}\right\} \quad(m \in \mathbb{N}) .
$$

Then, as $A\left(n_{0}\right)=\bigcup_{m \in \mathbb{N}} B(m)$, we deduce that $B\left(m_{0}\right)$ is uncountable for some $m_{0} \in \mathbb{N}$. Note that $\left\|x_{m_{0}}\right\| \geqslant 2 /\left(3 n_{0}\right)$. Now choose an integer $k$ such that 
$k>3 n_{0}\|T\|$, and take $k$ distinct ordinals $\alpha_{1}, \ldots, \alpha_{k} \in B\left(m_{0}\right)$. Since the function $\sum_{j=1}^{k} f_{\alpha_{j}} \in C_{0}\left[0, \omega_{1}\right)$ has norm one, we conclude that

$$
\|T\| \geqslant\left\|\sum_{j=1}^{k} T f_{\alpha_{j}}\right\| \geqslant k\left\|x_{m_{0}}\right\|-\sum_{j=1}^{k}\left\|x_{m_{0}}-T f_{\alpha_{j}}\right\| \geqslant \frac{k}{3 n_{0}}>\|T\|,
$$

which is clearly absurd.

For each countable ordinal $\alpha$, let $Q_{\alpha}$ be the canonical projection of $E_{\omega_{1}}$ onto the first $\alpha$ summands; that is, the $\beta^{\text {th }}$ coordinate of $Q_{\alpha}\left(f_{\gamma}\right)_{\gamma<\omega_{1}}$, where $\beta<\omega_{1}$ and $\left(f_{\gamma}\right)_{\gamma<\omega_{1}} \in E_{\omega_{1}}$, is given by $f_{\beta}$ if $\beta \leqslant \alpha$ and 0 otherwise. We then have the following counterpart of Lemma 2.7(ii).

Lemma 2.8. A subspace of $E_{\omega_{1}}$ is separable if and only if it is contained in the range of the projection $Q_{\alpha}$ for some countable ordinal $\alpha$.

Proof. The implication $\Leftarrow$ is clear.

Conversely, suppose that $W$ is a separable subspace of $E_{\omega_{1}}$, and let $D$ be a countable, dense subset of $W$. Since each element of $E_{\omega_{1}}$ has countable support, for each $x \in D$, we can choose a countable ordinal $\beta(x)$ such that $x=Q_{\beta(x)} x$. Then the ordinal $\alpha=\sup _{x \in D} \beta(x)$ is countable and satisfies $x=Q_{\alpha} x$ for each $x \in D$, and hence $W \subseteq Q_{\alpha}\left[E_{\omega_{1}}\right]$.

\section{Proofs of Theorem 1.1 and Corollaries 1.21 .3}

We are now ready to prove the results stated in Section 1 . We begin with a lemma which, in the light of Lemma 2.6(1), above, effectively establishes Theorem 1.1 in the case where $X=C_{0}\left[0, \omega_{1}\right)$ and $Y=C_{0}\left(L_{0}\right)$.

Lemma 3.1. Let $T: C_{0}\left[0, \omega_{1}\right) \rightarrow E_{\omega_{1}}$ be an operator with uncountable Szlenk index. Then:

(i) for each pair $(\alpha, \eta)$ of countable ordinals, there exist operators $R: C[0, \alpha] \rightarrow$ $C_{0}\left[0, \omega_{1}\right)$ and $S: E_{\omega_{1}} \rightarrow C[0, \alpha]$ and a countable ordinal $\xi>\eta$ such that the diagram

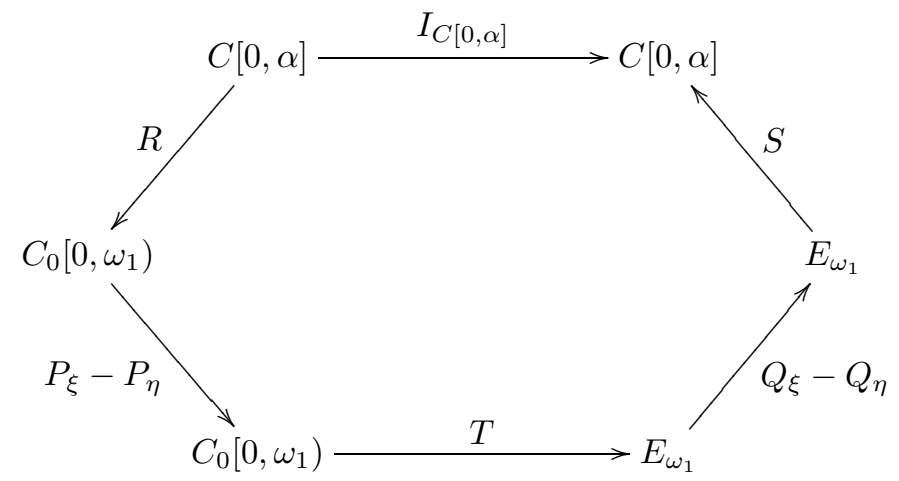

is commutative;

(ii) the identity operator on $E_{\omega_{1}}$ factors through $T$.

Proof. (ii) Let $U=\left(I_{E_{\omega_{1}}}-Q_{\eta}\right) T\left(I_{C_{0}\left[0, \omega_{1}\right)}-P_{\eta}\right)$. Since $T=U+Q_{\eta} T+T P_{\eta}-Q_{\eta} T P_{\eta}$, where each of the final three terms $Q_{\eta} T, T P_{\eta}$, and $Q_{\eta} T P_{\eta}$ has countable Szlenk index, Theorem 2.2 implies that $U$ has uncountable Szlenk index. Hence $U$ fixes an 
isomorphic copy of $C[0, \alpha]$ by Theorem 2.3 , that is, we can find a closed subspace $W$ of $C_{0}\left[0, \omega_{1}\right)$ such that $W$ is isomorphic to $C[0, \alpha]$ and the restriction of $U$ to $W$ is bounded below. Lemmas 2.7(ii) and 2.8 enable us to choose a countable ordinal $\xi>\eta$ such that the separable subspaces $W$ and $U[W]$ are contained in the ranges of the projections $P_{\xi}$ and $Q_{\xi}$, respectively. Then the restrictions to $W$ of the operators $U$ and $\left(Q_{\xi}-Q_{\eta}\right) T\left(P_{\xi}-P_{\eta}\right)$ are equal. By Theorem 2.5. $U[W]$ contains a closed subspace $Z$ which is isomorphic to $C[0, \alpha]$ and which is complemented in $Q_{\xi}\left[E_{\omega_{1}}\right]$, and therefore also in $E_{\omega_{1}}$. The conclusion now follows from Lemma 2.1 because $U$, and thus $\left(Q_{\xi}-Q_{\eta}\right) T\left(P_{\xi}-P_{\eta}\right)$, maps the closed subspace $W \cap U^{-1}[Z]$ isomorphically onto $Z$.

(ii) Using (ii) together with Lemmas 2.7(ii) and 2.8, we may inductively construct transfinite sequences of countable ordinals $(\eta(\alpha))_{\alpha<\omega_{1}}$ and $(\xi(\alpha))_{\alpha<\omega_{1}}$ and of operators $\left(R_{\alpha}: C[0, \alpha] \rightarrow C_{0}\left[0, \omega_{1}\right)\right)_{\alpha<\omega_{1}}$ and $\left(S_{\alpha}: E_{\omega_{1}} \rightarrow C[0, \alpha]\right)_{\alpha<\omega_{1}}$ such that $\eta(\alpha)<\xi(\alpha)<\eta(\beta)$,

$$
\begin{gathered}
I_{C[0, \alpha]}=S_{\alpha}\left(Q_{\xi(\alpha)}-Q_{\eta(\alpha)}\right) T\left(P_{\xi(\alpha)}-P_{\eta(\alpha)}\right) R_{\alpha}, \\
\left(I_{E_{\omega_{1}}}-Q_{\eta(\beta)}\right) T P_{\xi(\alpha)}=0, \quad \text { and } \quad Q_{\xi(\alpha)} T\left(I_{C_{0}\left[0, \omega_{1}\right)}-P_{\eta(\beta)}\right)=0
\end{gathered}
$$

whenever $0 \leqslant \alpha<\beta<\omega_{1}$. We may clearly also suppose that $\left\|R_{\alpha}\right\|=1$ for each $\alpha<\omega_{1}$.

Given $n \in \mathbb{N}$, set $A(n)=\left\{\alpha \in\left[0, \omega_{1}\right):\left\|S_{\alpha}\right\| \leqslant n\right\}$. Since $\left[0, \omega_{1}\right)=\bigcup_{n \in \mathbb{N}} A(n)$, we conclude that $A\left(n_{0}\right)$ is uncountable for some $n_{0} \in \mathbb{N}$. Then $E_{A\left(n_{0}\right)}$ is isomorphic to $E_{\omega_{1}}$ by Lemma 2.6(iii), so that it will suffice to show that the identity operator on $E_{A\left(n_{0}\right)}$ factors through $T$.

To this end, we observe that

$$
S: x \mapsto\left(S_{\alpha}\left(Q_{\xi(\alpha)}-Q_{\eta(\alpha)}\right) x\right)_{\alpha \in A\left(n_{0}\right)}, \quad E_{\omega_{1}} \rightarrow E_{A\left(n_{0}\right)},
$$

defines an operator of norm at most $n_{0}$. Moreover, introducing the subspaces

$$
F_{\beta}=\left\{\left(f_{\alpha}\right)_{\alpha \in A\left(n_{0}\right)} \in E_{A\left(n_{0}\right)}: f_{\alpha}=0(\alpha \neq \beta)\right\} \quad\left(\beta \in A\left(n_{0}\right)\right),
$$

we can define a linear contraction by

$$
R:\left(f_{\alpha}\right)_{\alpha \in A\left(n_{0}\right)} \mapsto \sum_{\alpha \in A\left(n_{0}\right)}\left(P_{\xi(\alpha)}-P_{\eta(\alpha)}\right) R_{\alpha} f_{\alpha}, \quad \text { span } \bigcup_{\beta \in A\left(n_{0}\right)} F_{\beta} \rightarrow C_{0}\left[0, \omega_{1}\right) .
$$

Since the domain of definition of $R$ is dense in $E_{A\left(n_{0}\right)}, R$ extends uniquely to a linear contraction defined on $E_{A\left(n_{0}\right)}$. Now, given $\beta \in A\left(n_{0}\right)$ and $\left(f_{\alpha}\right)_{\alpha \in A\left(n_{0}\right)} \in F_{\beta}$, we have

$\operatorname{STR}\left(f_{\alpha}\right)_{\alpha \in A\left(n_{0}\right)}=\left(S_{\alpha}\left(Q_{\xi(\alpha)}-Q_{\eta(\alpha)}\right) T\left(P_{\xi(\beta)}-P_{\eta(\beta)}\right) R_{\beta} f_{\beta}\right)_{\alpha \in A\left(n_{0}\right)}=\left(f_{\alpha}\right)_{\alpha \in A\left(n_{0}\right)}$

by (3.1) and the fact that $\left(Q_{\xi(\alpha)}-Q_{\eta(\alpha)}\right) T\left(P_{\xi(\beta)}-P_{\eta(\beta)}\right)=0$ for $\alpha \neq \beta$ by (3.2). This implies that $S T R=I_{E_{A\left(n_{0}\right)}}$, and the result follows.

Proof of Theorem 1.1 . The implications $(\underline{b}) \Rightarrow(\underline{a}) \Rightarrow(\mathbb{c})$ are clear, so it remains to prove that $(\mathrm{C}) \Rightarrow(\mathrm{b})$. Hence, we suppose that the Szlenk index of $T$ is uncountable, and seek to demonstrate that the identity operator on $C_{0}\left(L_{0}\right)$ factors through $T$. Throughout the proof, we shall tacitly use the fact that $C_{0}\left(L_{0}\right)$ is isomorphic to $E_{\omega_{1}}$; cf. Lemma 2.6(i).

Lemma 3.1(ii) establishes the desired conclusion for $X=C_{0}\left[0, \omega_{1}\right)$ and $Y=$ $C_{0}\left(L_{0}\right)$. 
Now suppose that $X=Y=C_{0}\left[0, \omega_{1}\right)$. If $T \notin \mathscr{M}$, then the identity operator on $C_{0}\left[0, \omega_{1}\right)$ factors through $T$ by Lemma $2.6(\nabla)$, and hence the identity operator on $C_{0}\left(L_{0}\right)$ also factors through $T$ by Lemma 2.6 (iv). Otherwise $T \in \mathscr{M}$, in which case condition (b) of Lemma 2.6(v) implies that $T=V U T$ for some operators $U: C_{0}\left[0, \omega_{1}\right) \rightarrow C_{0}\left(L_{0}\right)$ and $V: C_{0}\left(L_{0}\right) \rightarrow C_{0}\left[0, \omega_{1}\right)$. Then $U T$ has the same Szlenk index as $T$, so that, by the first case, the identity operator on $C_{0}\left(L_{0}\right)$ factors through $U T$, and hence through $T$.

Finally, suppose that $X=C_{0}\left(L_{0}\right)$, and either $Y=C_{0}\left[0, \omega_{1}\right)$ or $Y=C_{0}\left(L_{0}\right)$. By Lemma 2.6(iv), we can take operators $U: C_{0}\left(L_{0}\right) \rightarrow C_{0}\left[0, \omega_{1}\right)$ and $V: C_{0}\left[0, \omega_{1}\right) \rightarrow$ $C_{0}\left(L_{0}\right)$ such that $I_{C_{0}\left(L_{0}\right)}=V U$. Then $T V$ has the same Szlenk index as $T$, and the conclusion follows from the previous two cases, as above.

Proof of Corollary 1.2. The three sets in (1.2) are equal by the negation of Theorem 1.1. The final of these sets is clearly equal to $\bigcup_{\alpha<\omega_{1}} \mathscr{S} \mathscr{Z}_{\alpha}(X)$, which is an ideal of $\mathscr{B}(X)$ by Theorem 2.2 .

For $X=C_{0}\left(L_{0}\right)$, the fact that the second set in (1.2) is an ideal of $\mathscr{B}\left(C_{0}\left(L_{0}\right)\right)$ implies that it is necessarily the unique maximal ideal by an observation of Dosev and Johnson (see [10, p. 166]).

For $X=C_{0}\left[0, \omega_{1}\right)$, we have $\mathscr{S}_{C_{0}\left(L_{0}\right)}\left(C_{0}\left[0, \omega_{1}\right)\right) \varsubsetneqq \mathscr{M}$ because $\mathscr{S}_{C_{0}\left(L_{0}\right)}\left(C_{0}\left[0, \omega_{1}\right)\right)$ is an ideal of $\mathscr{B}\left(C_{0}\left[0, \omega_{1}\right)\right), \mathscr{M}$ is the unique maximal ideal, and any projection on $C_{0}\left[0, \omega_{1}\right)$ whose range is isomorphic to $C_{0}\left(L_{0}\right)$ is an example of an operator which belongs to $\mathscr{M} \backslash \mathscr{S}_{C_{0}\left(L_{0}\right)}\left(C_{0}\left[0, \omega_{1}\right)\right)$. To verify the final statement of the corollary, suppose that $\mathscr{I}$ is a proper ideal of $\mathscr{B}\left(C_{0}\left[0, \omega_{1}\right)\right)$ such that $\mathscr{I}$ is not contained in $\mathscr{S}_{C_{0}\left(L_{0}\right)}\left(C_{0}\left[0, \omega_{1}\right)\right)$. Then, by (1.2), $\mathscr{I}$ contains an operator $T$ such that $I_{C_{0}\left(L_{0}\right)}=$ $S T R$ for some operators $R: C_{0}\left(L_{0}\right) \rightarrow C_{0}\left[0, \omega_{1}\right)$ and $S: C_{0}\left[0, \omega_{1}\right) \rightarrow C_{0}\left(L_{0}\right)$. Given $U \in \mathscr{M}$, we can find operators $V: C_{0}\left[0, \omega_{1}\right) \rightarrow C_{0}\left(L_{0}\right)$ and $W: C_{0}\left(L_{0}\right) \rightarrow C_{0}\left[0, \omega_{1}\right)$ such that $U=W V$ by (1.1), and hence $U=(W S) T(R V) \in \mathscr{I}$. This proves that $\mathscr{M} \subseteq \mathscr{I}$, and consequently $\mathscr{M}=\mathscr{I}$.

Proof of Corollary 1.3. First, to show that $C_{0}\left(L_{0}\right)$ is primary, let $P \in \mathscr{B}\left(C_{0}\left(L_{0}\right)\right)$ be a projection. Since the ideal $\mathscr{S}_{C_{0}\left(L_{0}\right)}\left(C_{0}\left(L_{0}\right)\right)$ is proper, it cannot contain both $P$ and $I_{C_{0}\left(L_{0}\right)}-P$; we may without loss of generality suppose that $P \notin \mathscr{S}_{C_{0}\left(L_{0}\right)}\left(C_{0}\left(L_{0}\right)\right)$. Then, by (1.2),$I_{C_{0}\left(L_{0}\right)}$ factors through $P$, so that $P\left[C_{0}\left(L_{0}\right)\right]$ contains a closed, complemented subspace which is isomorphic to $C_{0}\left(L_{0}\right)$ by Lemma 2.1. Since $P\left[C_{0}\left(L_{0}\right)\right]$ is complemented in $C_{0}\left(L_{0}\right)$, and the Pełczyński decomposition method (as stated in [1, Theorem 2.23(b)], for instance) applies by Lemma 2.6(ii)-(ii), we conclude that $P\left[C_{0}\left(L_{0}\right)\right]$ and $C_{0}\left(L_{0}\right)$ are isomorphic, as desired.

Second, to verify that $C_{0}\left(L_{0}\right)$ is complementably homogeneous, suppose that $W$ is a closed subspace of $C_{0}\left(L_{0}\right)$ such that $W$ is isomorphic to $C_{0}\left(L_{0}\right)$. Take an isomorphism $U$ of $C_{0}\left(L_{0}\right)$ onto $W$, and let $J$ be the natural inclusion of $W$ into $C_{0}\left(L_{0}\right)$. Since the operator $J U \in \mathscr{B}\left(C_{0}\left(L_{0}\right)\right)$ fixes an isomorphic copy of $C_{0}\left(L_{0}\right)$, we can find operators $R$ and $S$ on $C_{0}\left(L_{0}\right)$ such that $I_{C_{0}\left(L_{0}\right)}=S(J U) R$ by Theorem 1.1 The operator $P=J U R S \in \mathscr{B}\left(C_{0}\left(L_{0}\right)\right)$ is then a projection, its range is clearly contained in $W$, and the restriction of $S$ to the range of $P$ is an isomorphism onto $C_{0}\left(L_{0}\right)$ (with inverse $J U R$ ).

\section{Closing Remarks}

The Banach algebras $\mathscr{B}\left(C_{0}\left[0, \omega_{1}\right)\right)$ and $\mathscr{B}\left(C\left[0, \omega_{1}\right]\right)$ are isomorphic because the underlying Banach spaces $C_{0}\left[0, \omega_{1}\right)$ and $C\left[0, \omega_{1}\right]$ are isomorphic. In particular, 
$\mathscr{B}\left(C_{0}\left[0, \omega_{1}\right)\right)$ and $\mathscr{B}\left(C\left[0, \omega_{1}\right]\right)$ have isomorphic lattices of closed ideals. The study of this lattice was initiated in [14, while Corollary 1.2 of the present paper adds another element to our understanding of it. Figure 1 summarizes our current knowledge of this lattice, extending [14, Figure 1].

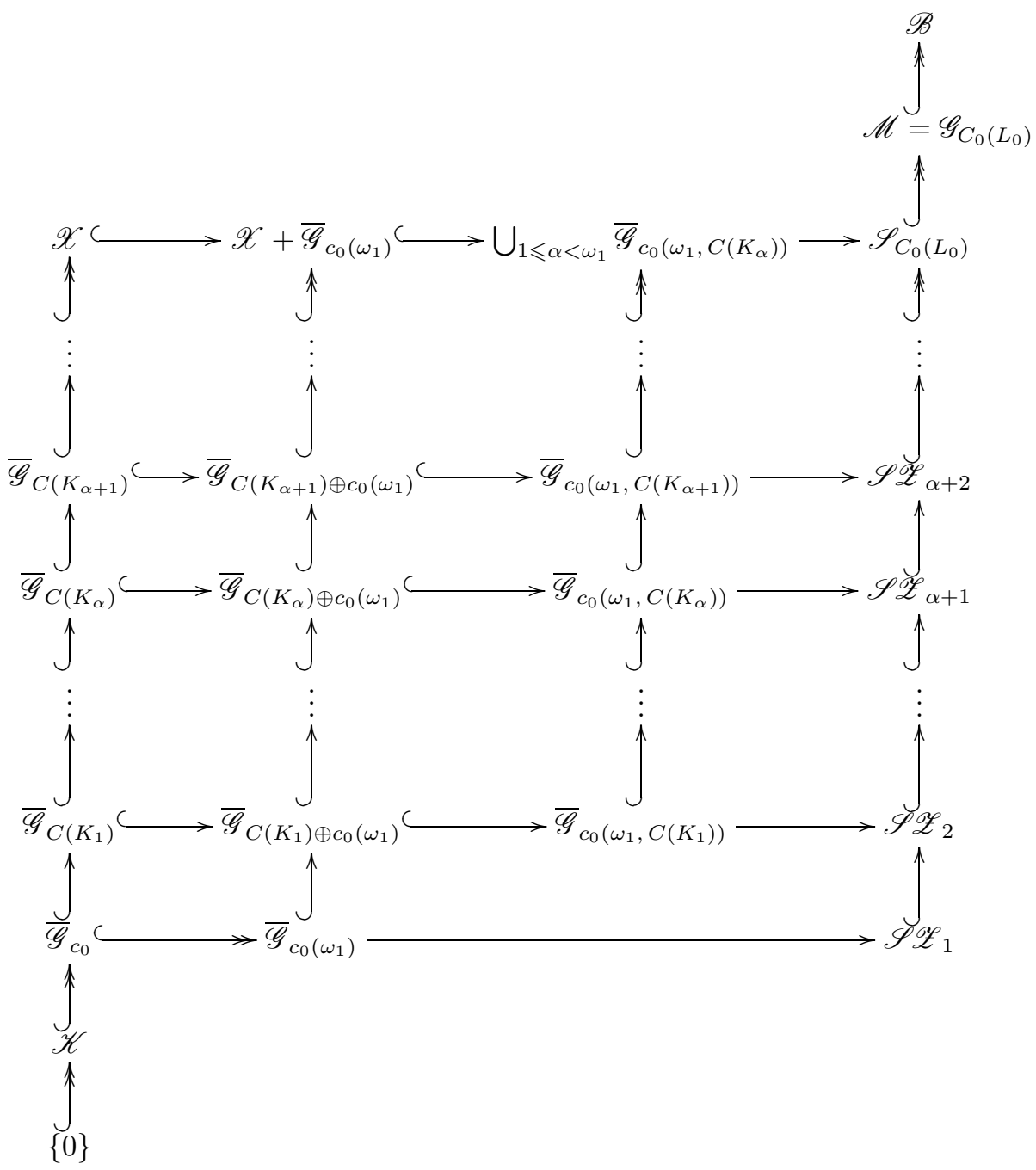

Figure 1. Partial structure of the lattice of closed ideals of $\mathscr{B}=\mathscr{B}\left(C_{0}\left[0, \omega_{1}\right)\right)$.

In addition to the notation that has already been introduced, Figure 1 relies on the following conventions: the Banach space $C_{0}\left[0, \omega_{1}\right)$ is suppressed everywhere, so that we write $\mathscr{B}$ instead of $\mathscr{B}\left(C_{0}\left[0, \omega_{1}\right)\right)$, etc.; $\mathscr{K}$ denotes the ideal of compact operators, $\mathscr{X}$ denotes the ideal of operators with separable range, $\mathscr{G}_{X}$ denotes the ideal of operators that factor through the Banach space $X$, and $\overline{\mathscr{G}}_{X}$ denotes its closure in the operator norm; $c_{0}\left(\omega_{1}, X\right)$ denotes the $c_{0}$-direct sum of $\omega_{1}$ copies of the Banach space $X$; we write $c_{0}\left(\omega_{1}\right)$ in the case where $X$ is the scalar field; $\mathscr{I} \longrightarrow \mathscr{J}$ signifies that the ideal $\mathscr{I}$ is contained in the ideal $\mathscr{J}, \mathscr{I} \hookrightarrow \mathscr{J}$ 
indicates that the inclusion is proper, while $\mathscr{I} \hookrightarrow \mathscr{J}$ means that there are no closed ideals between $\mathscr{I}$ and $\mathscr{J}$; and finally $K_{\alpha}=\left[0, \omega^{\omega^{\alpha}}\right]$, where $\alpha$ is a countable ordinal.

The relations among the ideals in the first and second columns of Figure 1 were all established in [14, Section 5]. The double-headed arrows at the top of these columns are meant to signify that

$$
\mathscr{X}\left(C_{0}\left[0, \omega_{1}\right)\right)=\bigcup_{\alpha<\omega_{1}} \overline{\mathscr{G}}_{C\left(K_{\alpha}\right)}\left(C_{0}\left[0, \omega_{1}\right)\right)
$$

and

$$
\left(\mathscr{X}+\overline{\mathscr{G}}_{c_{0}\left(\omega_{1}\right)}\right)\left(C_{0}\left[0, \omega_{1}\right)\right)=\bigcup_{\alpha<\omega_{1}} \overline{\mathscr{G}}_{C\left(K_{\alpha}\right) \oplus c_{0}\left(\omega_{1}\right)}\left(C_{0}\left[0, \omega_{1}\right)\right),
$$

respectively. Indeed, (4.1) is immediate from Lemma 2.7(1) (which shows that it is in fact not necessary to take the closure of the ideals $\mathscr{G}_{C\left(K_{\alpha}\right)}\left(C_{0}\left[0, \omega_{1}\right)\right)$ on the right-hand side), and (4.2) can easily be verified using (4.1) together with the fact that the ideal $\left(\mathscr{X}+\overline{\mathscr{G}}_{c_{0}\left(\omega_{1}\right)}\right)\left(C_{0}\left[0, \omega_{1}\right)\right)$ is closed by (the easy first part of) [14, Theorem 5.8]. The counterpart of (4.1)-(4.2) for the third column is obvious, while (1.2) establishes the corresponding identity for the final column.

We shall next show that

$$
\overline{\mathscr{G}}_{C\left(K_{\alpha}\right) \oplus c_{0}\left(\omega_{1}\right)}\left(C_{0}\left[0, \omega_{1}\right)\right) \varsubsetneqq \overline{\mathscr{G}}_{c_{0}\left(\omega_{1}, C\left(K_{\alpha}\right)\right)}\left(C_{0}\left[0, \omega_{1}\right)\right) \subseteq \mathscr{S} \mathscr{Z}_{\alpha+1}\left(C_{0}\left[0, \omega_{1}\right)\right)
$$

for each countable ordinal $\alpha \geqslant 1$. The first inclusion is immediate because $c_{0}\left(\omega_{1}, C\left(K_{\alpha}\right)\right)$ contains a closed, complemented subspace which is isomorphic to $C\left(K_{\alpha}\right) \oplus c_{0}\left(\omega_{1}\right)$, while the second follows from [7. Theorem 2.12], which states that (the identity operator on) the Banach space $c_{0}\left(\omega_{1}, C\left(K_{\alpha}\right)\right)$ has the same Szlenk index as $C\left(K_{\alpha}\right)$, that is, $\omega^{\alpha+1}$, so that $I_{c_{0}\left(\omega_{1}, C\left(K_{\alpha}\right)\right)}$ belongs to $\mathscr{S}_{\mathscr{Z}}{ }_{\alpha+1}$, and this operator ideal is closed. To see that the first inclusion in (4.3) is proper, we assume the contrary and seek a contradiction. The assumption implies that there exists an isomorphism $U$ of $C\left(K_{\alpha}\right) \oplus c_{0}\left(\omega_{1}\right)$ onto $c_{0}\left(\omega_{1}, C\left(K_{\alpha}\right)\right)$ (see, e.g., [5, Lemma 3.1]). Denote by $P \in \mathscr{B}\left(C\left(K_{\alpha}\right) \oplus c_{0}\left(\omega_{1}\right)\right)$ the canonical projection with range $C\left(K_{\alpha}\right)$ and kernel $c_{0}\left(\omega_{1}\right)$. Then $P$ belongs to $\mathscr{X}\left(C\left(K_{\alpha}\right) \oplus c_{0}\left(\omega_{1}\right)\right)$ and $I_{C\left(K_{\alpha}\right) \oplus c_{0}\left(\omega_{1}\right)}-P$ belongs to $\mathscr{S} \mathscr{Z}_{1}\left(C\left(K_{\alpha}\right) \oplus c_{0}\left(\omega_{1}\right)\right)$, so that $Q=U P U^{-1}$ belongs to $\mathscr{X}\left(c_{0}\left(\omega_{1}, C\left(K_{\alpha}\right)\right)\right)$, while $I_{c_{0}\left(\omega_{1}, C\left(K_{\alpha}\right)\right)}-Q$ belongs to $\mathscr{S}_{\mathcal{Z}}{ }_{1}\left(c_{0}\left(\omega_{1}, C\left(K_{\alpha}\right)\right)\right)$. Arguing as in the proof of Lemma 2.8, we see that since the range of $Q$ is separable, it is contained in the range of the canonical projection $Q_{\beta}$ of $c_{0}\left(\omega_{1}, C\left(K_{\alpha}\right)\right)$ onto the first $\beta$ summands for some countable ordinal $\beta$. Consequently, we have

$$
\left(I_{c_{0}\left(\omega_{1}, C\left(K_{\alpha}\right)\right)}-Q_{\beta}\right)\left(I_{c_{0}\left(\omega_{1}, C\left(K_{\alpha}\right)\right)}-Q\right)=I_{c_{0}\left(\omega_{1}, C\left(K_{\alpha}\right)\right)}-Q_{\beta},
$$

where the operator on the left-hand side has Szlenk index (at most) $\omega$, while the operator on the right-hand side has Szlenk index $\omega^{\alpha+1}$. This is clearly impossible, and the proof of (4.3) is therefore complete.

We do not know whether the second inclusion in (4.3) is proper. Since this appears to be an interesting question, we shall raise it formally.

Question 4.1. Are the ideals $\overline{\mathscr{G}}_{c_{0}\left(\omega_{1}, C\left(K_{\alpha}\right)\right)}\left(C_{0}\left[0, \omega_{1}\right)\right)$ and $\mathscr{S} \mathscr{Z}_{\alpha+1}\left(C_{0}\left[0, \omega_{1}\right)\right)$ equal for some, or possibly all, countable ordinals $\alpha \geqslant 1$ ?

It is important that the underlying Banach space is $C_{0}\left[0, \omega_{1}\right)$ in this question; indeed, it is easy to find an example of a Banach space $X$ on which there is an 
operator $T$ which has Szlenk index at most $\omega^{\alpha+1}$, but $T$ does not factor approximately through $c_{0}\left(\omega_{1}, C\left(K_{\alpha}\right)\right)$. For instance, take $X=\ell_{2}$ and $T=I_{X}$; then the Szlenk index of $T$ is $\omega$, but $T$ does not factor approximately through $c_{0}\left(\omega_{1}, C\left(K_{\alpha}\right)\right)$ because no closed (complemented) subspace of $c_{0}\left(\omega_{1}, C\left(K_{\alpha}\right)\right)$ is isomorphic to an infinite-dimensional Hilbert space.

Finally, to see that the inclusions

$$
\overline{\mathscr{G}}_{c_{0}\left(\omega_{1}, C\left(K_{\alpha}\right)\right)}\left(C_{0}\left[0, \omega_{1}\right)\right) \subseteq \overline{\mathscr{G}}_{c_{0}\left(\omega_{1}, C\left(K_{\alpha+1}\right)\right)}\left(C_{0}\left[0, \omega_{1}\right)\right)
$$

and $\mathscr{S} \mathscr{Z}_{\alpha+1}\left(C_{0}\left[0, \omega_{1}\right)\right) \subseteq \mathscr{S} \mathscr{Z}_{\alpha+2}\left(C_{0}\left[0, \omega_{1}\right)\right)$ are proper for each countable ordinal $\alpha \geqslant 1$, we observe that, for $\beta=\omega^{\omega^{\alpha+1}}$, the projection $P_{\beta}$ defined immediately before Lemma 2.7 belongs to $\mathscr{G}_{C\left(K_{\alpha+1}\right)}\left(C_{0}\left[0, \omega_{1}\right)\right) \backslash \mathscr{S} \mathscr{Z}_{\alpha+1}\left(C_{0}\left[0, \omega_{1}\right)\right)$.

One may wonder whether it is possible to obtain a complete description of the lattice of closed ideals of $\mathscr{B}\left(C_{0}\left[0, \omega_{1}\right)\right)$. As explained in [14, p. 4834], we consider this an extremely difficult problem because "in the first instance, one would need to classify the closed ideals of $\mathscr{B}\left(C\left[0, \omega^{\omega^{\alpha}}\right]\right)$ for each countable ordinal $\alpha$, something that already appears intractable; it has currently been achieved only in the simplest case $\alpha=0$, where $C[0, \omega] \cong c_{0}$."

\section{REFERENCES}

[1] Fernando Albiac and Nigel J. Kalton, Topics in Banach space theory, Graduate Texts in Mathematics, vol. 233, Springer, New York, 2006. MR2192298(2006h:46005)

[2] Dale E. Alspach, $C(K)$ norming subsets of $C[0,1]$, Studia Math. 70 (1981), no. 1, 27-61. MR646959 (83h:46032)

[3] D. Alspach and Y. Benyamini, Primariness of spaces of continuous functions on ordinals, Israel J. Math. 27 (1977), no. 1, 64-92. MR.0440349 (55 \#13224)

[4] C. Bessaga and A. Pełczyński, Spaces of continuous functions. IV. On isomorphical classification of spaces of continuous functions, Studia Math. 19 (1960), 53-62. MR.0113132 (22 \#3971)

[5] Alistair Bird, Graham Jameson, and Niels Jakob Laustsen, The Giesy-James theorem for general index $p$, with an application to operator ideals on the pth James space, J. Operator Theory 70 (2013), no. 1, 291-307, DOI 10.7900/jot.2011aug11.1936. MR3085829

[6] J. Bourgain, The Szlenk index and operators on $C(K)$-spaces, Bull. Soc. Math. Belg. Sér. B 31 (1979), no. 1, 87-117. MR.592664 (83j:46027)

[7] Philip A. H. Brooker, Direct sums and the Szlenk index, J. Funct. Anal. 260 (2011), no. 8, 2222-2246, DOI 10.1016/j.jfa.2010.12.016. MR2772370(2012f:46024)

[8] Philip A. H. Brooker, Asplund operators and the Szlenk index, J. Operator Theory 68 (2012), no. 2, 405-442. MR2995728

[9] John B. Conway, A course in functional analysis, 2nd ed., Graduate Texts in Mathematics, vol. 96, Springer-Verlag, New York, 1990. MR1070713 (91e:46001)

[10] D. Dosev and W. B. Johnson, Commutators on $\ell_{\infty}$, Bull. Lond. Math. Soc. 42 (2010), no. 1, 155-169, DOI 10.1112/blms/bdp110. MR2586976 (2011d:47084)

[11] Petr Hájek and Gilles Lancien, Various slicing indices on Banach spaces, Mediterr. J. Math. 4 (2007), no. 2, 179-190, DOI 10.1007/s00009-007-0111-4. MR2340479(2008f:46022)

[12] Petr Hájek, Vicente Montesinos Santalucía, Jon Vanderwerff, and Václav Zizler, Biorthogonal systems in Banach spaces, CMS Books in Mathematics/Ouvrages de Mathématiques de la SMC, 26, Springer, New York, 2008. MR2359536 (2008k:46002)

[13] Tomasz Kania, Piotr Koszmider, and Niels Jakob Laustsen, A weak*-topological dichotomy with applications in operator theory, Trans. London Math. Soc. 1 (2014), no. 1, 1-28. MR.3247078

[14] Tomasz Kania and Niels Jakob Laustsen, Uniqueness of the maximal ideal of the Banach algebra of bounded operators on $C\left(\left[0, \omega_{1}\right]\right)$, J. Funct. Anal. 262 (2012), no. 11, 4831-4850, DOI 10.1016/j.jfa.2012.03.011. MR.2913688

[15] A. Pełczyński, On C (S)-subspaces of separable Banach spaces, Studia Math. 31 (1968), 513522. MR0234261 (38 \#2578) 
[16] C. Samuel, Indice de Szlenk des $C(K)$ (K espace topologique compact dénombrable) (French), Seminar on the geometry of Banach spaces, Vol. I, II (Paris, 1983), Publ. Math. Univ. Paris VII, vol. 18, Univ. Paris VII, Paris, 1984, pp. 81-91. MR781569 (87b:46026)

[17] W. Szlenk, The non-existence of a separable reflexive Banach space universal for all separable reflexive Banach spaces, Studia Math. 30 (1968), 53-61. MR0227743 (37 \#3327)

Department of Mathematics and Statistics, Fylde College, Lancaster University, Lancaster LA1 4YF, United Kingdom - and - Institute of Mathematics, Polish Academy of Sciences, ul. Śniadeckich 8, 00-956 Warszawa, Poland

E-mail address: tomasz.marcin.kania@gmail.com

Department of Mathematics and Statistics, Fylde College, Lancaster University, LANCASTER LA1 4YF, United Kingdom

E-mail address: n.laustsen@lancaster.ac.uk 